\title{
Study on GPS/INS System Using Novel Filtering Methods for Vessel Attitude Determination
}

\author{
Xiyuan Chen, Chong Shen, and Yuefang Zhao \\ Key Laboratory of Micro-Inertial Instrument and Advanced Navigation Technology, Ministry of Education and School of \\ Instrument Science and Engineering, Southeast University, Nanjing 210096, China \\ Correspondence should be addressed to Xiyuan Chen; chxiyuan@seu.edu.cn
}

Received 22 February 2013; Accepted 26 March 2013

Academic Editor: Henryk Śniegocki

Copyright (C) 2013 Xiyuan Chen et al. This is an open access article distributed under the Creative Commons Attribution License, which permits unrestricted use, distribution, and reproduction in any medium, provided the original work is properly cited.

\begin{abstract}
Any vehicle such as vessel has three attitude parameters, which are mostly defined as pitch, roll, and heading from true north. In hydrographic surveying, determination of these parameters by using GPS or INS technologies is essential for the requirements of vehicle measurements. Recently, integration of GPS/INS by using data fusion algorithm became more and more popular. Therefore, the data fusion algorithm plays an important role in vehicle attitude determination. To improve attitude determination accuracy and efficiency, two improved data fusion algorithms are presented, which are extended Kalman particle filter (EKPF) and genetic particle filter (GPF). EKPF algorithm combines particle filter (PF) with the extended Kalman filter (EKF) to avoid sample impoverishment during the resampling process. GPF is based on genetic algorithm and PF; several genetic operators such as selection, crossover, and mutation are adopted to optimize the resampling process of PF, which can not only reduce the particle impoverishment but also improve the computation efficiency. The performances of the system based on the two proposed algorithms are analyzed and compared with traditional KF. Simulation results show that, comprehensively considering the determination accuracy and consumption cost, the performance of the proposed GPF is better than EKPF and traditional KF.
\end{abstract}

\section{Introduction}

It is very important to provide accurate and reliable attitude determination data since the performance of a vessel is highly reliant on the attitude determination system [1]. Due to their complementary features of global positioning system (GPS) and inertial navigation system (INS), the GPS/INS integrated navigation systems have been extensively investigated and increasingly used for highly accurate attitude determination [2], especially in position and orientation systems [3]. The GPS/INS integrated systems can be classified into loosely coupled, tightly coupled, and ultratightly coupled systems [4]. Due to the high cost and complexity of tightly-coupled and ultra-tightly-coupled systems, most GPS/INS systems are loosely-coupled because they are easier to build [5]. Therefore, loosely-coupled GPS/INS is employed in our study. The block diagram of loosely-coupled GPS/INS is shown in Figure 1.

In the process of attitude determination, filtering method performs a very important role to achieve high accuracy determination results with high efficiency [6-8]. Kalman filter (KF) [9-13] is a popular data fusion algorithm in handling optimal estimation problems which has been widely investigated in vehicle positioning or attitude determination for a long time. However, the optimality of KF highly depends on the data linearity. The first solution to the estimation of nonlinear system is extended Kalman filter (EKF) [1417]. The EKF is based on approximation to the first-order linearization of the nonlinear process or observation equations, so large errors can be introduced which make EKF algorithm no longer effective in many special applications, such as the complex systems environments which demand higher estimation accuracy. Recently, unscented Kalman filter (UKF) $[18,19]$ and particle filter (PF) [20-22] are presented through improvement and innovation. Compared with traditional filtering algorithms, $\mathrm{PF}$ is more suitable for non-Gaussian and nonlinear systems. However, because of the so-called sample impoverishment phenomenon, the implementation complexity of PF is prohibitively high with 


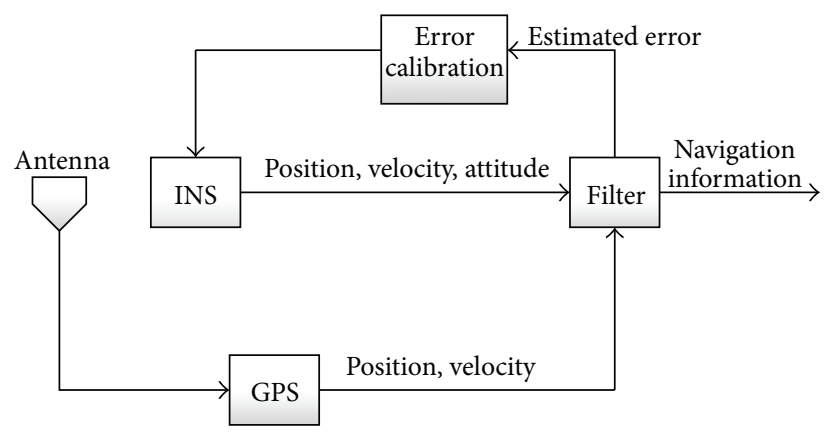

FIGURE 1: Block diagram of loosely-coupled GPS/INS system.

limited computing resources, especially for dynamical systems. So the improvement of PF has been concerned by many scholars for a long time. Extended Kalman particle filter (EKPF) [23], which uses EKF to determine the importance density, has been proposed to improve the performance of PF. In addition, with the development of simulated annealing algorithm, genetic algorithm [24], particle swarm optimization [25], and some other intelligent evolutionary algorithms
[26] have been used to improve the diversity of particle groups for accessing to the global optimum estimation, and certain effects have been reached indeed through the improvements above.

The major contributions of this paper are in investigating the EKPF and genetic particle filter (GPF) and using them to improve not only the accuracy of GPS/INS attitude determination but also the computation efficiency. The simulation results show that, compared with traditional KF, more highly accurate attitude determination results can be reached by using EKPF. The comparison results between EKPF and GPF show that GPF has higher filtering accuracy and more efficient computation ability than EKPF.

\section{System Model and Data Collection}

2.1. System Model. The state model for KF is presented as

$$
\dot{X}(t)=F(t) X(t)+G(t) W(t),
$$

where $X$ denotes state variables, $W$ denotes system noise, $F$ denotes system matrix, and $G$ denotes system noise matrix. The state vector is parameters errors with 15 dimensions:

$$
X=\left[\begin{array}{lllllllllllllll}
\delta L & \delta \lambda & \delta h & \delta V_{E} & \delta V_{N} & \delta V_{U} & \phi_{E} & \phi_{N} & \phi_{U} & \nabla_{b x} & \nabla_{b y} & \nabla_{b z} & \varepsilon_{b x} & \varepsilon_{b y} & \varepsilon_{b z}
\end{array}\right],
$$

where, $\delta L, \delta \lambda, \delta h, \delta V_{E}, \delta V_{N}, \delta V_{U}, \phi_{E}, \phi_{N}, \phi_{U}$ are, respectively, the 3-dimension position errors, velocity errors and attitude errors of INS system. The 9 parameters mentioned above are estimated by filter, then the determination accuracy can be calibrated by the compensation of the 9 estimated parameters, and the measurement accuracy can be improved after calibrating. $\nabla_{b x}, \nabla_{b y}, \nabla_{b z}, \varepsilon_{b x}, \varepsilon_{b y}, \varepsilon_{b z}$ are, respectively, the 3-dimension errors of accelerometers and gyros. The 6 parameters mentioned above are determined by experience or device introduction, and the accuracy of GPS/INS system can be improved by putting the 6 parameters into the state vector. The system noise matrix is

$$
W=\left[\begin{array}{lllllllllllllll}
w_{L} & w_{\lambda} & w_{h} & w_{V_{E}} & w_{V_{N}} & w_{V_{U}} & w_{\phi_{E}} & w_{\phi_{N}} & w_{\phi_{U}} & 0 & 0 & 0 & 0 & 0 & 0
\end{array}\right],
$$

where $w_{L}, w_{\lambda}, w_{h}, w_{V_{E}}, w_{V_{N}}, w_{V_{U}}, w_{\phi_{E}}, w_{\phi_{N}}, w_{\phi_{U}}$ are, respectively, the stochastic noise of 3-dimension position, velocity, and attitude errors.

The following are the measured state variables:

$$
\begin{gathered}
\delta V_{E}=V_{E}^{\mathrm{INS}}-V_{E}^{\mathrm{GPS}}, \\
\delta V_{N}=V_{N}^{\mathrm{INS}}-V_{N}^{\mathrm{GPS}}, \\
\delta V_{U}=V_{U}^{\mathrm{INS}}-V_{U}^{\mathrm{GPS}}, \\
\delta L=L^{\mathrm{INS}}-L^{\mathrm{GPS}}, \\
\delta \lambda=\lambda^{\mathrm{INS}}-\lambda^{\mathrm{GPS}}, \\
\delta h=h^{\mathrm{INS}}-h^{\mathrm{GPS}} .
\end{gathered}
$$

The measurement model is defined as

$$
\begin{gathered}
Z(t)=H(t) X(t)+V(t) \\
Z=\left[\begin{array}{llllll}
\delta L & \delta \lambda & \delta h & \delta V_{E} & \delta V_{N} & \delta V_{U}
\end{array}\right] .
\end{gathered}
$$

2.2. Data Collection. A set of vessel dynamic data are selected to verify the proposed filtering algorithms. The experiment parameters are as follows: gyro constant drifts of east, north, and up direction are all $1^{\circ} / \mathrm{h}$; gyro random drift is $0.3^{\circ} / \mathrm{h}$; accelerometer constant and random biases both are $0.08 \mathrm{mg}$; original heading, pitch, and roll angle are, respectively, $79.36^{\circ}$, $0^{\circ}$, and $0^{\circ}$; original velocity is $0.01 \mathrm{~m} / \mathrm{s}$ and original velocity error is $0.01 \mathrm{~m} / \mathrm{s}$; and original longitude is $126.682234^{\circ}$ and original latitude is $45.776563^{\circ}$. The GPS/INS system is shown in Figure 2. The INS and GPS data acquisition interfaces in $\mathrm{PC}$ are shown in Figures 3 and 4, respectively. 


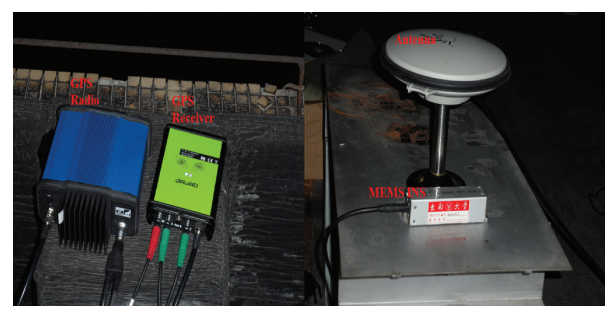

Figure 2: The GPS/INS system.

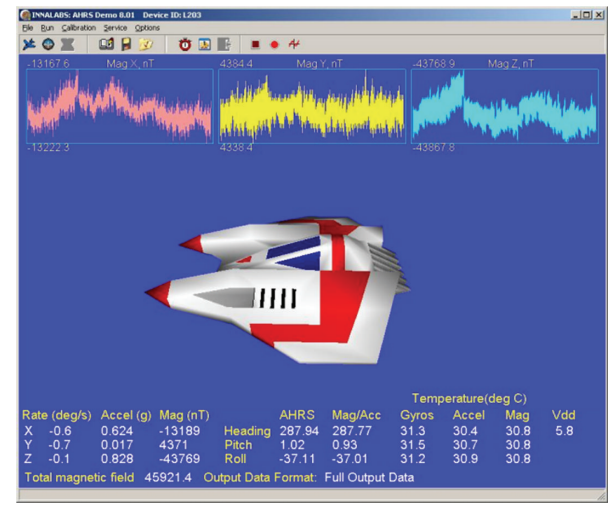

FIgURE 3: The INS data acquisition interface in PC.

\section{Extended Kalman Particle Filter}

3.1. Traditional Kalman Filter. The Kalman filter is a set of mathematical equations which can provide an efficient computational method to recursively estimate the state and error covariance of a process, in a way that minimizes the mean of the squared error covariance. The estimate process contains two steps: prediction and update. Consider a state space dynamic equation of a time-variant system model and a measurement model as

$$
\begin{gathered}
X_{k}=\phi_{k, k-1} X_{k-1}+\Gamma_{k-1} W_{k-1}, \\
Z_{k}=H_{k} X_{k-1}+V_{k},
\end{gathered}
$$

where the subscript $k$ stands for the iteration time $t_{k} ; X_{k}$ is the state of system at time $t_{k-1} ; Z_{k}$ is the measurement at time $t_{k} ; \phi$ and $H$ are the state and measurement transition matrices, respectively, which in practice can be considered as invariable; $W_{k}$ denotes the process noise; $V_{k}$ denotes the measurement noise; and $\Gamma$ denotes the process noise matrices.

The equations for the KF are divided into two groups: time update equations and measurement update equations. The time update equations also can be thought of as predictor equations, while the measurement update equations can be thought of as corrector equations. If $\phi, H$, and $Q$ can be assumed as constant, the time update equations are as follows:

$$
\text { Predicted state: } \widehat{X}_{k \mid k-1}=\phi_{k, k-1} \widehat{X}_{k-1} \text {, }
$$

Prediction covariance: $P_{k \mid k-1}=\phi_{k, k-1} P_{k-1} \phi_{k, k-1}^{T}+\Gamma_{k-1} Q_{k-1} \Gamma_{k-1}^{T}$,

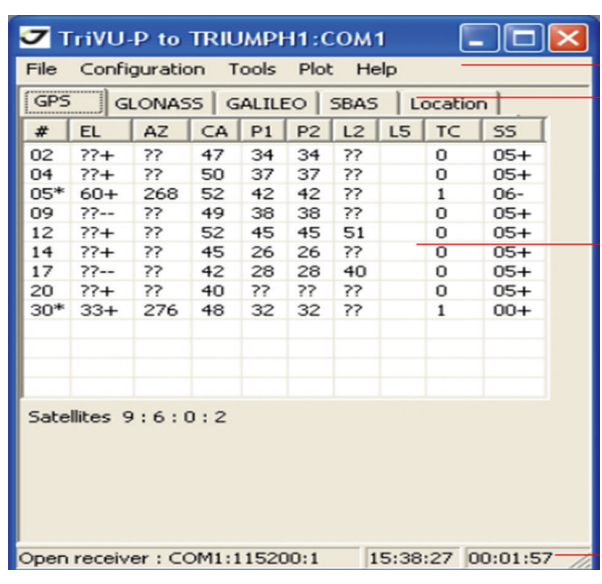

FIgURE 4: The GPS data acquisition interface in PC.

TABLE 1: Comparisons of consumption burden.

\begin{tabular}{lcc}
\hline & KF & EKPF \\
\hline Time $(\mu \mathrm{s})$ & 423.20 & 1095.98 \\
\hline
\end{tabular}

where $Q$ is the process noise covariance matrix. While the measurement update equations are

$$
\begin{aligned}
& \text { Kalman gain: } K_{k}=P_{k \mid k-1} H_{k}^{T}\left(H_{k} P_{k \mid k-1} H_{k}^{T}+R_{k}\right)^{-1}, \\
& \text { Estimated state: } X_{k}=\widehat{X}_{k \mid k-1}+K_{k}\left(Z_{k}-H_{k} \widehat{X}_{k \mid k-1}\right) \\
& \text { Estimated covariance: } P_{k}=\left(I-K_{k} H_{k}\right) P_{k \mid k-1} .
\end{aligned}
$$

3.2. Extended Kalman Particle Filter. PF algorithm is based on sequential importance sampling (SIS) step, which forms the basis for most sequential Monte Carlo filters. Compared with the traditional $\mathrm{KF}, \mathrm{PF}$ is more suitable for nonlinear and non-Gaussian systems, so PF is gradually used in signal tracking, robot control, navigation and positioning, and many other fields. But it also has many drawbacks, such as degeneracy phenomenon, large amount of computation, and sample impoverishment caused by re-sampling. A more detailed description of PF is beyond the scope of this paper. The reader is encouraged to consult one of the many papers about the PF, such as [20-22].

In order to improve the performance of PF, EKF algorithm is introduced. It uses the local linearization method for approximation of the importance density moving the particles to the high likelihood region, and then the optimal importance density can be approximated. This filtering algorithm is called EKPF. The EKPF algorithm is summarized as follows.

(i) Initialization:

$$
X_{k} \sim p\left(x_{0}\right), \quad \widehat{P}_{0}^{i}=\operatorname{var}\left(x_{0}\right), \quad w_{0}^{i}=\frac{1}{N} .
$$


(ii) Update the particle by EKF: $\left[\left\{X_{k}^{i}, \widehat{P}_{k}^{i}\right\}_{i=1}^{N}\right]=\operatorname{EKF}\left[\left\{X_{k-1}^{i}\right.\right.$, $\left.\widehat{P}_{k-1}^{i}\right\}_{i=1}^{N}, Z_{k}$, the specific steps as follows:

$$
\begin{gathered}
\widehat{X}_{k \mid k-1}^{i}=f\left(X_{k-1}^{i}\right), \\
P_{k \mid k-1}^{i}=F_{k}^{i} P_{k-1}\left(F_{k}^{i}\right)^{T}+F_{k}^{i} Q_{k}\left(F_{k}^{i}\right)^{T}, \\
K_{k}=P_{k \mid k-1}^{i}\left(H_{k}^{i}\right)^{T}\left[R_{k}+H_{k}^{i} P_{k \mid k-1}^{i}\left(H_{k}^{i}\right)^{T}\right]^{-1}, \\
\widehat{X}_{k}^{i}=\widehat{X}_{k \mid k-1}^{i}+K_{k}\left(Z_{k}-h\left(\widehat{X}_{k \mid k-1}^{i}\right)\right), \\
\widehat{P}_{k}^{i}=P_{k \mid k-1}^{i}-K_{k} H_{k}^{i} P_{k \mid k-1}^{i} .
\end{gathered}
$$

(iii) Particle importance weight after updating:

$$
\begin{aligned}
& X_{k}^{i} \sim q\left(\widehat{X}_{k}^{i} \mid X_{k-1}^{i}, Z_{k}\right)=N\left(\widehat{X}_{k}^{i}, \widehat{P}_{k}^{i}\right), \\
& \widehat{w}_{k}^{i}=w_{k-1}^{i} \frac{p\left(Z_{k} \mid \widehat{X}_{k}^{i}\right) p\left(\widehat{X}_{k}^{i} \mid X_{k-1}^{i}\right)}{q\left(\widehat{X}_{k}^{i} \mid X_{k-1}^{i}, Z_{1: k}\right)} .
\end{aligned}
$$

(iv) Normalize the importance weights:

$$
\widetilde{w}_{k}^{i}=\widehat{w}_{k}^{i} \cdot\left(\sum_{j=1}^{N} \widehat{w}_{k}^{j}\right)^{-1} .
$$

(v) State estimation:

$$
\widehat{X}_{k}=\frac{1}{N} \sum_{i=1}^{N} \widetilde{w}_{k}^{i} X_{k}^{i} .
$$

(vi) Re-sampling:

$$
\left[\left\{X_{k}^{i}, \widetilde{w}_{k}^{i}\right\}_{i=1}^{N}\right]=\text { Resample }\left[\left\{X_{k}^{i}, \widetilde{w}_{k}^{i}\right\}_{i=1}^{N}\right]
$$

The flow diagram of EKPF is shown as Figure 5.

3.3. Comparison Results. Figures 6, 7, and 8 show the performances comparison between $\mathrm{KF}$ and EKPF algorithms. Data are collected during 3200 seconds. In order to evaluate the performances of different algorithms, standard deviation of errors (SDE) is introduced. Figure 9 is the comparison of SDE; it can be concluded that EKPF performs much better than KF in filtering accuracy.

Another evaluation criterion of algorithm performance is computation burden. In this paper, the calculation time of each output is used as evaluation indicator, which is shown in Table 1. From Table 1 we can see that due to the complexity of EKPF algorithm, the computation consumption is highly increased from $423.2 \mu$ s to $1095.98 \mu$ s. Therefore, comprehensively considering the filtering accuracy and instantaneity, it can be concluded that EKPF is not the most available algorithm. In order to take both filtering accuracy and computation burden into consideration, the combination of genetic algorithm and particle filter is proposed in Section 4.

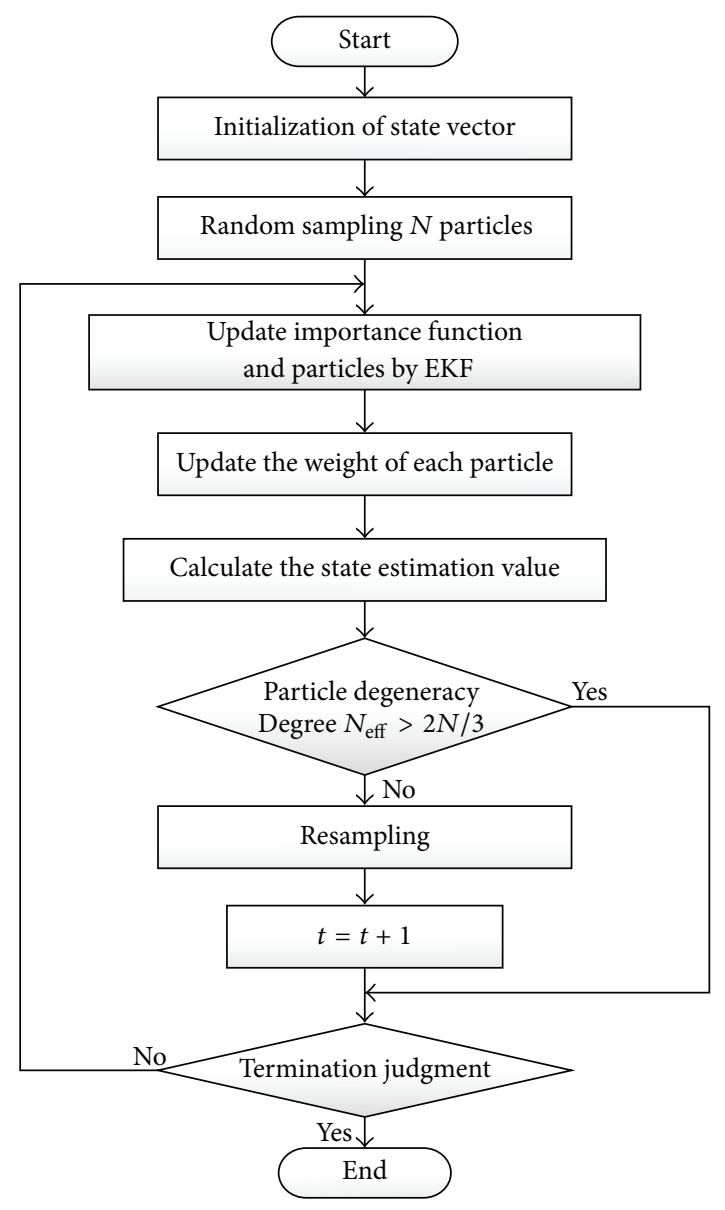

FIGURE 5: The flow diagram of EKPF.

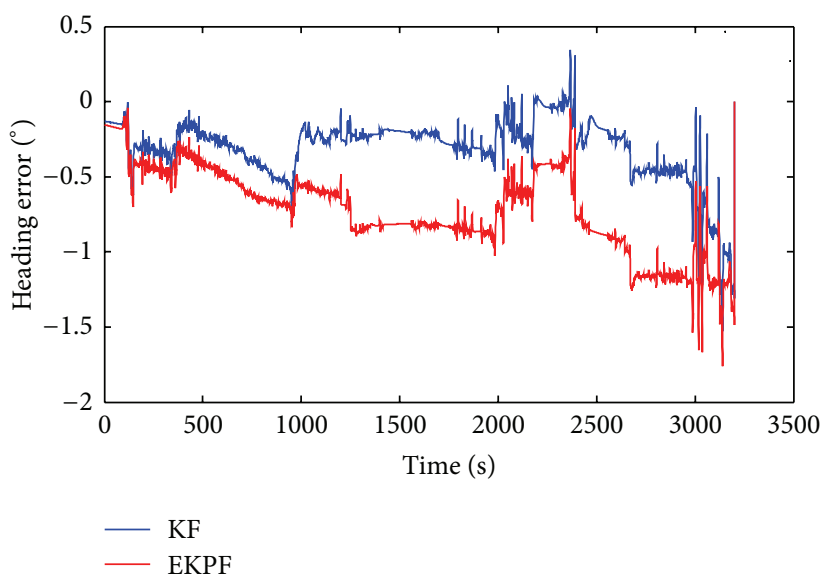

FIGURE 6: Filtering results of heading angle by KF and EKPF.

\section{Genetic Particle Filter}

4.1. Algorithms. Genetic algorithms (GAs) are search methods based on the mechanization of natural selection and natural genetics. GAs combine survival of the fittest among string structures (chromosomes) with randomized information exchange. A simple GA consists of three stages: 
for from 1 to $N_{1}$

for from 1 to $N$

find particle $X_{k}^{i}$ with the biggest weight $w_{k}^{i}$ from $N$ particles $* /$ This is the best particle; end

find particle $X_{k}^{j}$ with the biggest weight $w_{k}^{j}$ from $N$ particles $* /$ This is the worst particle;

$\widetilde{X}_{k}^{j}=a * X_{k}^{i}+(1-a) X_{k}^{j} * /$ Replace the worst particle by the linear combination of $X_{k}^{i}$ and $X_{k}^{j}, 0 \leq a \leq 1$; end Re-calculate the weight of $\widetilde{X}_{k}^{j}$ and then normalized it;

Algorithm 1

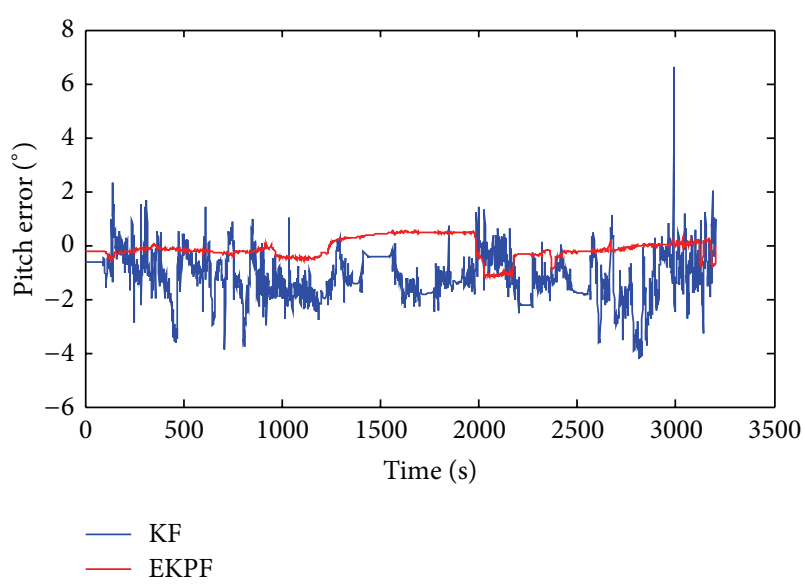

FIGURE 7: Filtering results of pitch angle by KF and EKPF.

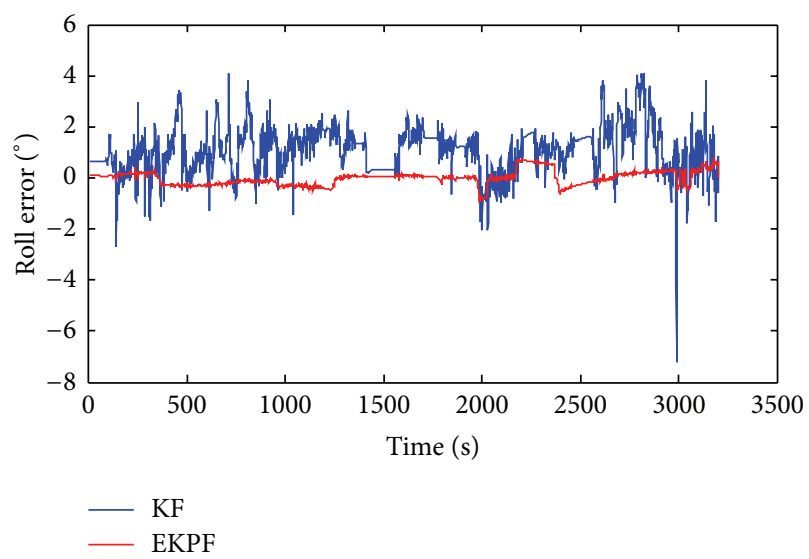

FIGURE 8: Filtering results of roll angle by KF and EKPF.

selection, crossover, and mutation. Selection is the process in which individual chromosomes are being selected according to their fitness function. By this process, the more likely chromosomes will contribute offspring in the next generation with higher probability. Crossover is the process that changing genetic information between two reproduced chromosomes occurs in. Even though the population can be improved by reproduction and crossover process, they can become overzealous and lose potentially important genetic information. Mutation process can protect against such an

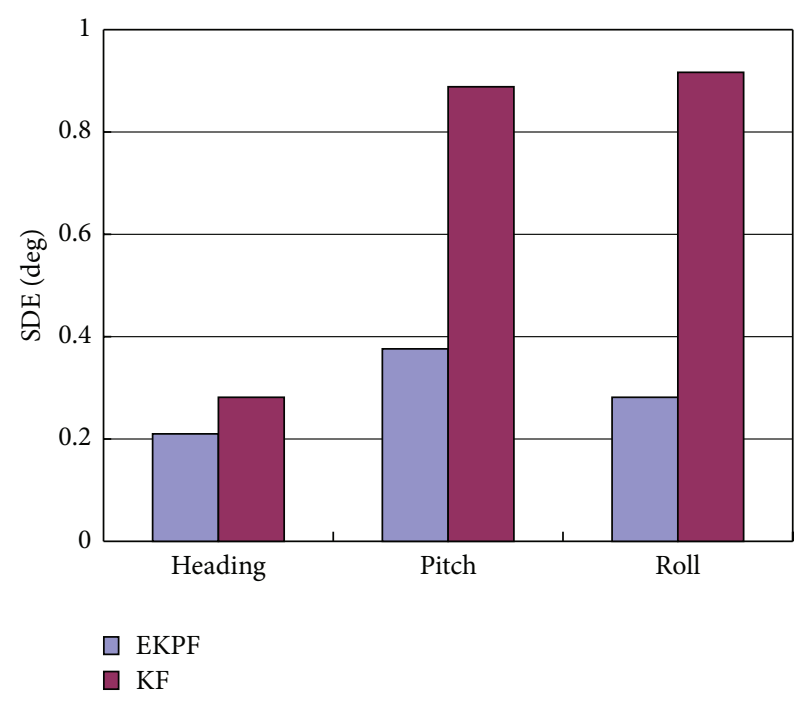

FIgURE 9: Comparisons of standard deviations.

irrecoverable loss by simply altering a character with small probability every once in a while.

Aiming to solve the particle degeneracy phenomenon existing in the standard PF algorithm, GPF uses genetic algorithm as the re-sampling method of $\mathrm{PF}$ algorithm. The genetic selection, crossover, and mutation operations are introduced into GPF to improve the re-sampling process, which can not only reduce the particle degeneracy phenomenon but also decrease the computation time. The key point of GPF is using GA to improve the re-sampling process of PF; the diagram is shown in Figure 10.

Here we only discuss the genetic algorithm scheme of resampling process. The details can be seen as follows.

(i) Genetic Crossover Operation

Processing the selected particles by crossover algorithm, assume that the number of particles which need to be crossover is $N_{1}$, then the implementation process can be shown as in Algorithm 1 .

(ii) Genetic Mutation Operation

The equation of mutation operation is

$$
X_{k}^{j}=\widetilde{X}_{k}^{j}+\eta,
$$

where, $\eta \in N(0, \Sigma)$. 


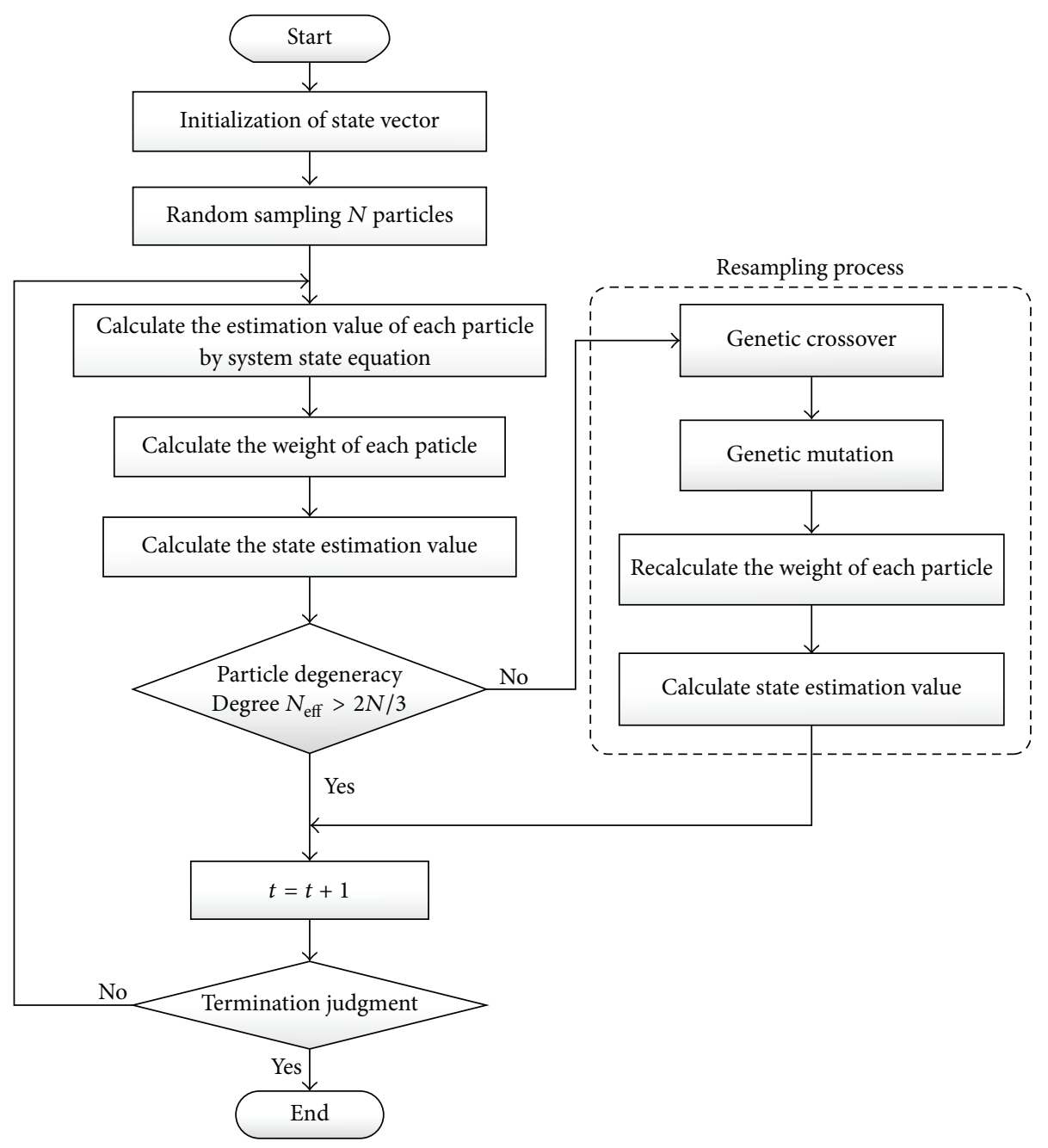

FIgURE 10: The flow diagram of GPF.

TABLE 2: Comparisons of consumption burden.

\begin{tabular}{lcc}
\hline & EKPF & GPF \\
\hline Time $(\mu \mathrm{s})$ & 1095.98 & 786.53 \\
\hline
\end{tabular}

After GA re-sampling, the state and variance can be estimated as

$$
\begin{gathered}
\widehat{X}_{k}=\sum_{i=1}^{N} w_{k}^{i} X_{k}^{i}, \\
P_{k}=\sum_{i=1}^{N} w_{k}^{i}\left(X_{k}^{i}-\widehat{X}_{k}\right)\left(X_{k}^{i}-\widehat{X}_{k}\right)^{T} .
\end{gathered}
$$

4.2. Comparison Results. Figures 11,12 , and 13 show the performances comparison between EKPF and GPF algorithms. From the comparison results we can see that all the heading, pitch, and roll errors are decreased by using GPF algorithm. Figure 14 also proves that GPF has better performance in filtering than EKPF since the SDEs are reduced effectively.

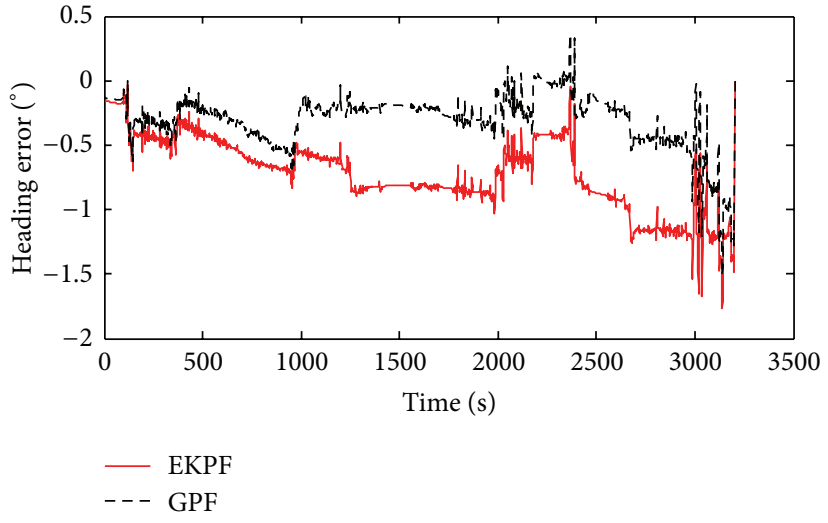

FIGURE 11: Filtering results of heading angle by EKPF and GPF.

Table 2 is the comparison results of consumption burden; compared to EKPF, the calculation time of GPF is significantly decreased. The calculation time of GPF is still longer than $\mathrm{KF}$, but comprehensively considering the filtering 


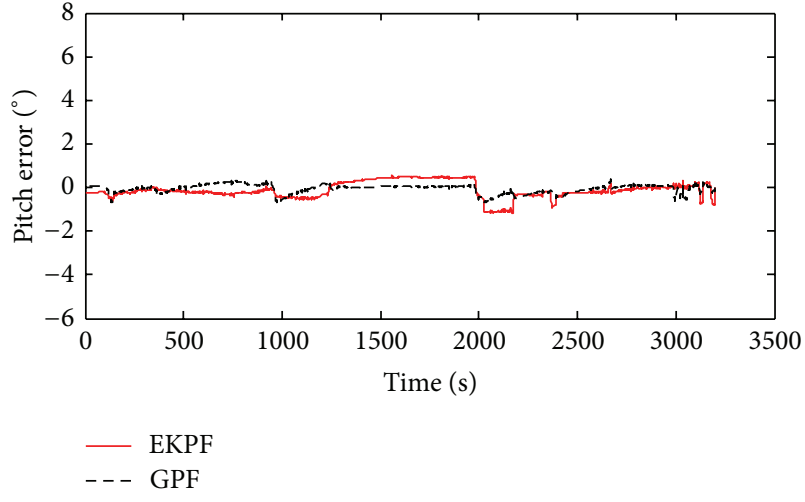

FIGURE 12: Filtering results of pitch angle by EKPF and GPF.

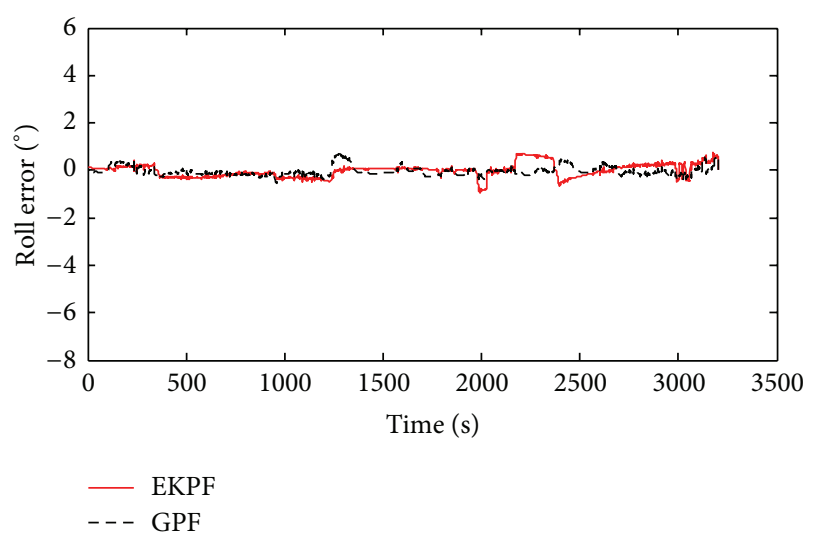

FIGURE 13: Filtering results of roll angle by EKPF and GPF.

accuracy and computation burden, it can be concluded that GPF is the most available filtering algorithm among the three algorithms mentioned above.

\section{Conclusions}

The problem of choosing a suitable filter for attitude determination application is studied here. Due to the low filtering accuracy of KF and the particle degeneracy phenomenon of $\mathrm{PF}$, two improved filters are presented in this paper, which are EKPF and GPF, respectively. And then the three filtering methods (KF, EKPF, and GPF) for attitude determination using GPS/INS system are studied, and their performances are compared.

The presented algorithms are tested with vessel attitude data, and the simulation results demonstrate that GPF yields the best accuracy under the same condition. In addition, the computation cost of the three filtering methods is analyzed in this paper; it shows that KF requires the lowest computation time, while EKPF requires the largest computation time. Comprehensively considering the filtering accuracy and computation cost, it can be concluded that the GPF is the most available filter among the three presented filtering methods.

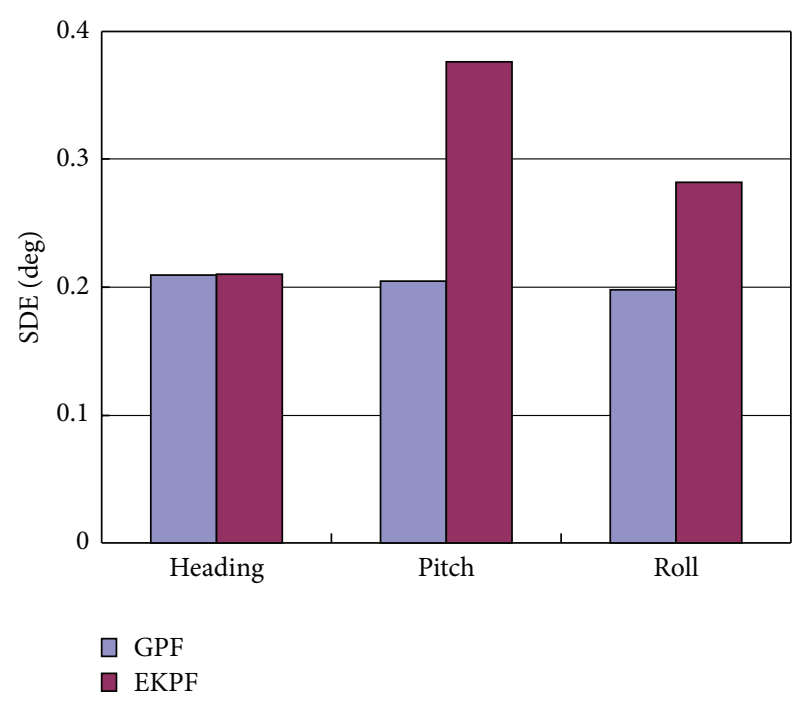

FIGURE 14: Comparisons of standard deviations.

\section{Acknowledgments}

This work is supported by Ocean special funds for scientific research on public causes (no. 201205035-09), Specialized Research Fund for the Doctoral Program of Higher Education (no. 20110092110039), National Natural Science Foundation of China (no. 50975049), 973 Program (no. 2009CB724002), Research Innovation Program for College Graduates of Jiangsu Province (no. CXZZ_0144), and The Scientific Research Foundation of Graduate School of Southeast University (no. ybjj1130).

\section{References}

[1] R. M. Alkan and O. Baykal, "Survey boat attitude determination with GPS/IMU systems," Journal of Navigation, vol. 54, no. 1, pp. 135-144, 2001.

[2] Y. Li, M. Efatmaneshnik, and A. G. Dempster, "Attitude determination by integration of MEMS inertial sensors and GPS for autonomous agriculture applications," GPS Solutions, vol. 16, no. 1, pp. 41-52, 2012.

[3] J. L. Li, J. C. Fang, and S. S. Ge, "Kinetics and design of a mechanically dithered ring laser gyroscope position and orientation system," IEEE Transactions on Instrumentation and Measurement, vol. 62, pp. 210-220, 2013.

[4] D. B. Hwang, D. W. Lim, S. L. Cho, and S. J. Lee, "Unified approach to ultra-tightly-coupled GPS/INS integrated navigation system," IEEE Aerospace and Electronic Systems Magazine, vol. 26, no. 3, pp. 30-38, 2011.

[5] D. T. Knight, "Rapid development of tightly-coupled GPS/INS systems," IEEE Aerospace and Electronic Systems Magazine, vol. 12, no. 2, pp. 14-18, 1997.

[6] K. Xiong, L. Liu, and Y. Liu, "Non-linear robust filter design for satellite attitude determination," IET Control Theory and Applications, vol. 4, no. 7, pp. 1222-1234, 2010.

[7] Y. Xu, X. Y. Chen, and Q. H. Li, "INS/WSN-Integrated navigation utilizing LS-SVM and H-infinity filtering," Mathematical Problems in Engineering, vol. 2012, pp. 1-19, 2012. 
[8] A. Nemra and N. Aouf, "Robust INS/GPS sensor fusion for UAV localization using SDRE nonlinear filtering," IEEE Sensors Journal, vol. 10, no. 4, pp. 789-798, 2010.

[9] S. H. P. Won, W. Melek, and F. Golnaraghi, "Fastening tool tracking system using a Kalman filter and particle filter combination," Measurement Science and Technology, vol. 12, pp. 1-12, 2011.

[10] D. Y. Kim and M. Jeon, "Robust distributed Kalman filter for wireless sensor networks with uncertain communication channels," Mathematical Problems in Engineering, vol. 2012, Article ID 238597, 12 pages, 2012.

[11] X. J. Sun, Y. Gao, Z. L. Deng, C. Li, and J. W. Wang, "Multimodel information fusion Kalman filtering and white noise deconvolution," Information Fusion, vol. 11, no. 2, pp. 163-173, 2010.

[12] X. L. Ning, X. Ma, C. Peng, W. Quan, and J. C. Fang, "Analysis of filtering methods for satellite autonomous orbit determination using celestial and geomagnetic measurement," Mathematical Problems in Engineering, vol. 2012, Article ID 267875, 16 pages, 2012.

[13] S. Wang, C. M. Wang, M. L. Chang, C. T. Tsai, and C. I. Chang, "Applications of kalman filtering to single hyperspectral signature analysis," IEEE Sensors Journal, vol. 10, pp. 547-563, 2010.

[14] F. Jiancheng and Y. Sheng, "Study on innovation adaptive EKF for in-flight alignment of airborne POS," IEEE Transactions on Instrumentation and Measurement, vol. 60, no. 4, pp. 1378-1388, 2011.

[15] C. S. Draper, J. F. Mahfouf, and J. P. Walker, "An EKF assimilation of AMSR-E soil moisture into the ISBA land surface scheme," Journal of Geophysical Research D, vol. 114, no. 20, Article ID D20104, 2009.

[16] G. P. Huang, A. I. Mourikis, and S. I. Roumeliotis, "Observability-based rules for designing consistent EKF SLAM estimators," International Journal of Robotics Research, vol. 29, no. 5, pp. 502-528, 2010.

[17] L. F. De Melo and J. F. Mangili Jr., "Trajectory planning for nonholonomic mobile robot using extended Kalman filter," Mathematical Problems in Engineering, vol. 2010, Article ID 979205, 22 pages, 2010.

[18] D. J. Jwo and C. N. Lai, "Unscented Kalman filter with nonlinear dynamic process modeling for GPS navigation," GPS Solutions, vol. 12, no. 4, pp. 249-260, 2008.

[19] U. Z. Ijaz, S. I. Chung, A. K. Khambampati, K. Y. Kim, and S. Kim, "Electrical resistance imaging of a time-varying interface in stratified flows using an unscented Kalman filter," Measurement Science and Technology, vol. 19, no. 6, Article ID 065501, 2008.

[20] S. Särkkä, A. Vehtari, and J. Lampinen, "Rao-Blackwellized particle filter for multiple target tracking," Information Fusion, vol. 8 , no. 1, pp. 2-15, 2007.

[21] S. Hong, J. Lee, A. Athalye, P. M. Djurić, and W. D. Cho, "Design methodology for domain specific parameterizable particle filter realizations," IEEE Transactions on Circuits and Systems I, vol. 54, no. 9, pp. 1987-2000, 2007.

[22] X. H. Zhong, A. B. Premkumar, and A. S. Madhukumar, "Particle filtering and posterior cramer-rao bound for 2-D direction of arrival tracking using an acoustic vector sensor," IEEE Sensors Journal, vol. 12, pp. 363-377, 2012.

[23] H. Qin, L. Cong, and Z. Li, "Resampling from the Niching genetic algorithm applicated in Extended Kalman particle filter," Chinese Journal of Electronics, vol. 20, no. 3, pp. 553-559, 2011.

[24] C. Perales-Graván and R. Lahoz-Beltra, "An AM radio receiver designed with a genetic algorithm based on a bacterial conjugation genetic operator," IEEE Transactions on Evolutionary Computation, vol. 12, no. 2, pp. 129-142, 2008.

[25] Y. Fu, M. Ding, and C. Zhou, "Phase angle-encoded and quantum-behaved particle swarm optimization applied to three-dimensional route planning for UAV,' IEEE Transactions on Systems, Man, and Cybernetics A, vol. 42, no. 2, pp. 511-526, 2012.

[26] X. Ning and J. Fang, "Spacecraft autonomous navigation using unscented particle filter-based celestial/Doppler information fusion," Measurement Science and Technology, vol. 19, no. 9, Article ID 095203, 2008. 


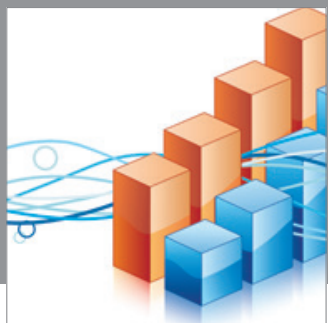

Advances in

Operations Research

mansans

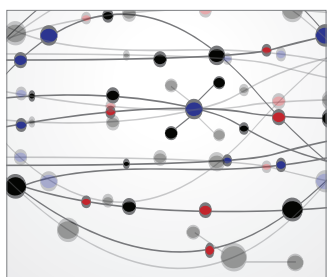

The Scientific World Journal
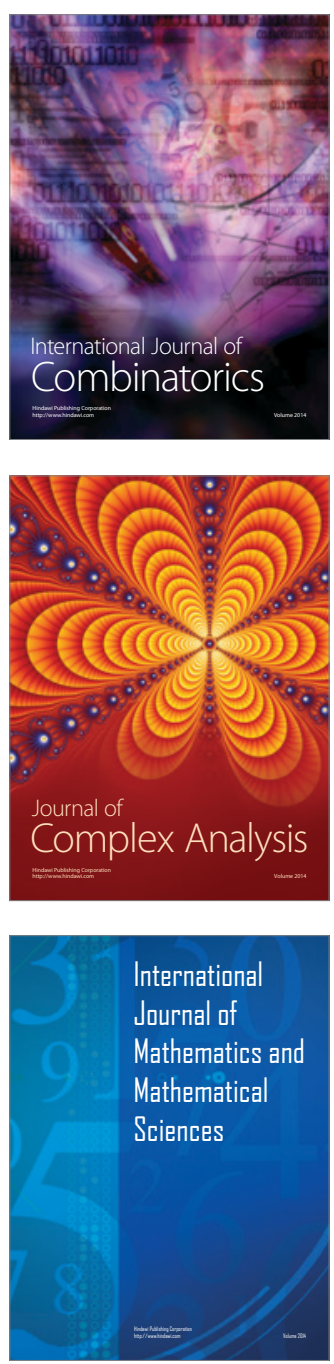
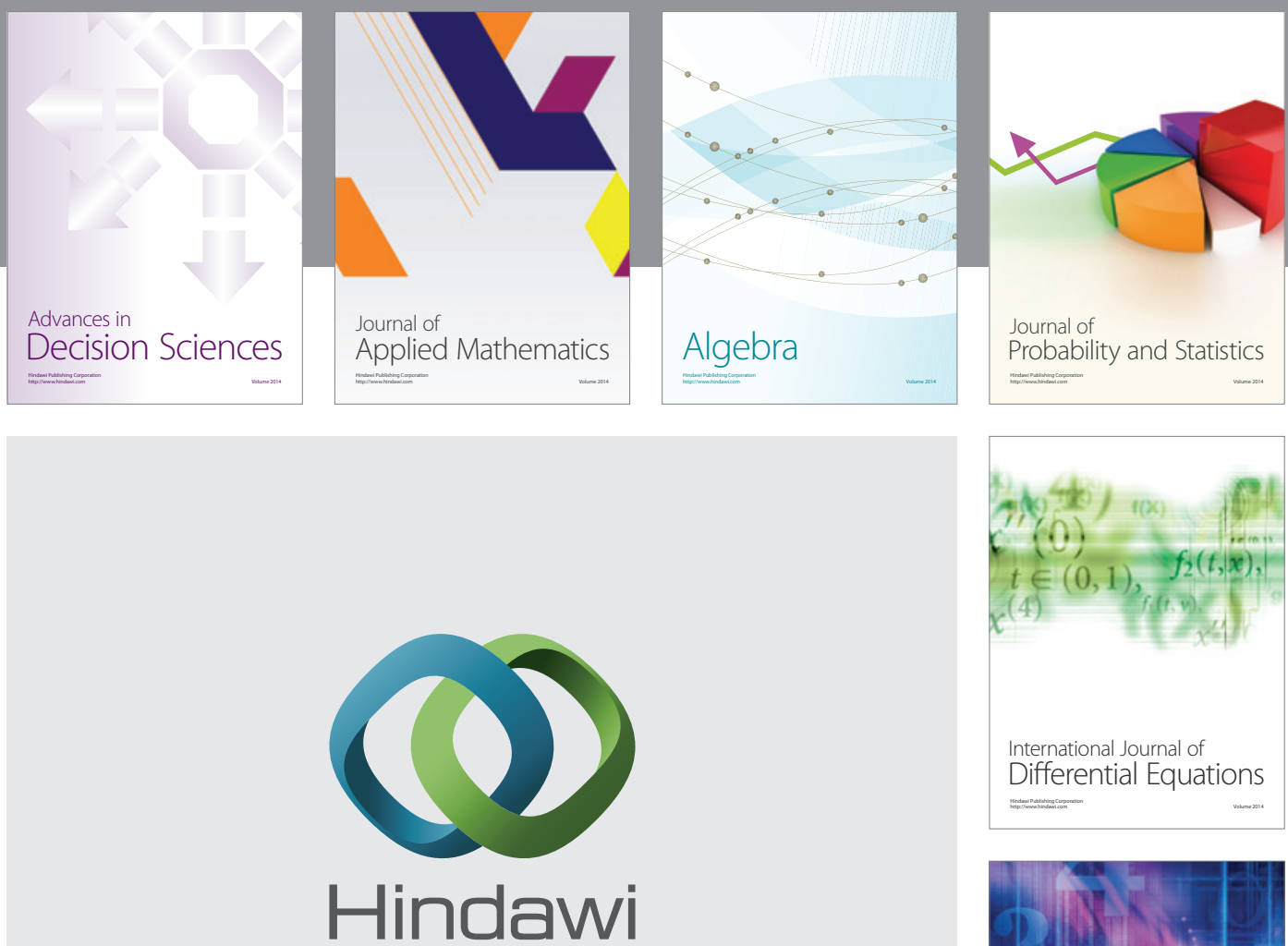

Submit your manuscripts at http://www.hindawi.com
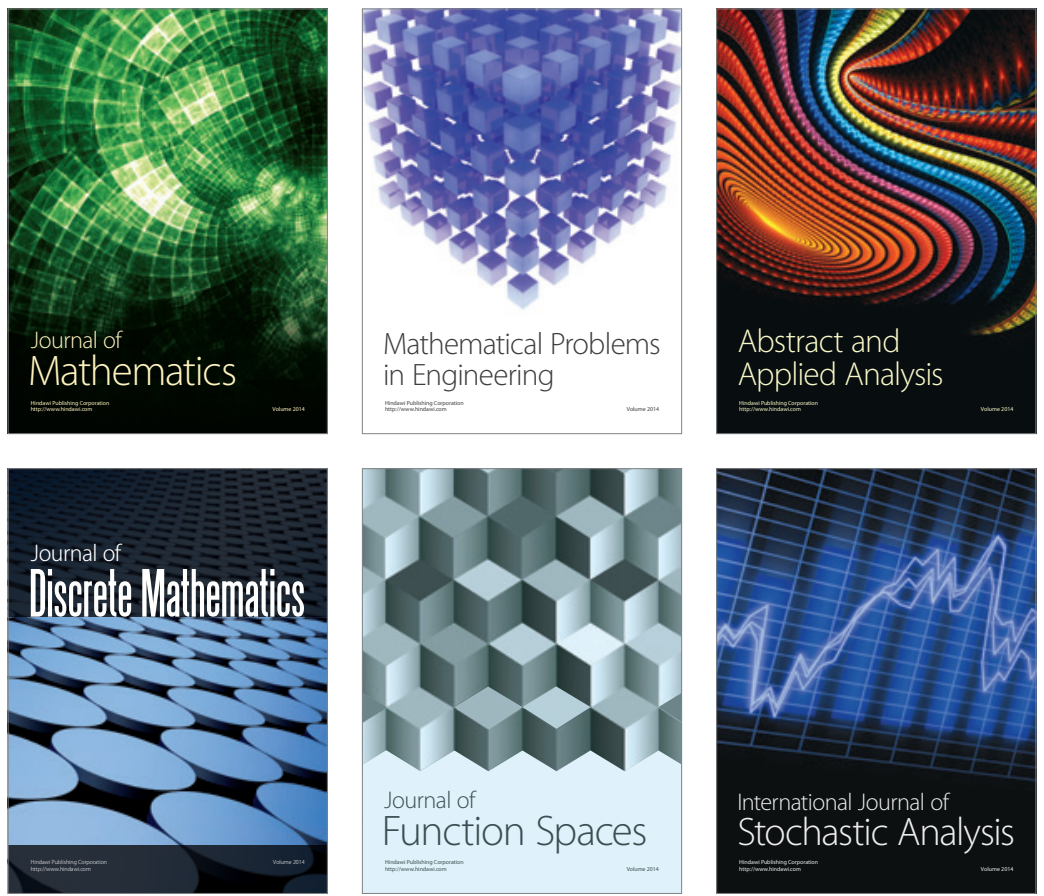

Journal of

Function Spaces

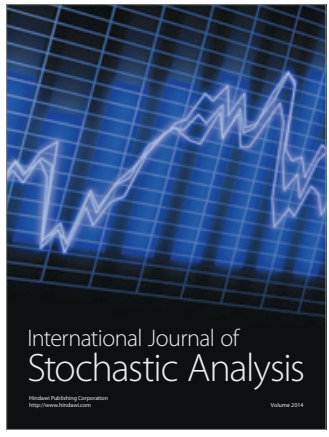

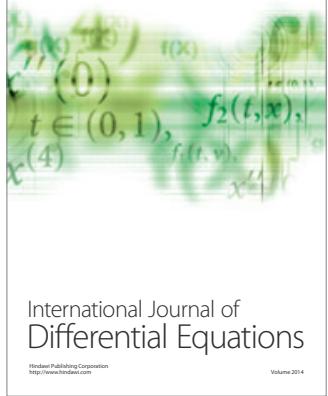
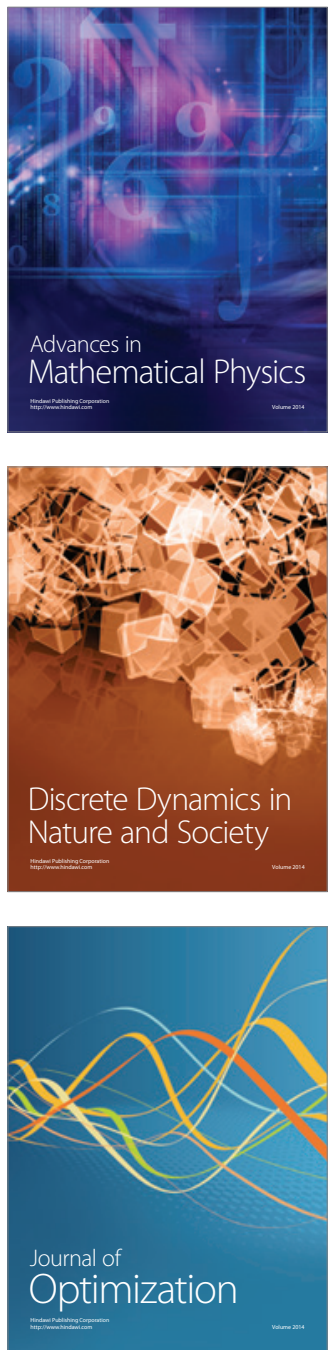